
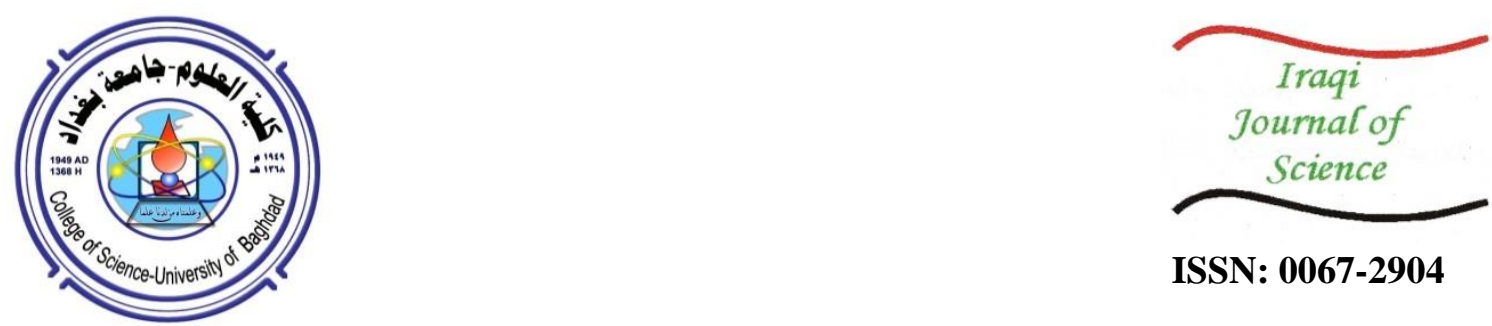

ISSN: 0067-2904

\title{
Detection of Chlamydia Pneumoniae in Lung Tissues Derived from Lung Tumor- Bearing Iraqi Patients
}

\author{
Zainab M. Lateef ${ }^{* 1}$, Alice K. Melconian, Ehab D. Salman \\ Department of Biotechnology, College of Sciences, University of Baghdad
}

Received: $19 / 11 / 2020$

Accepted: 5/2/2021

\begin{abstract}
Chlamydia pneumoniae is an intracellular gram-negative bacteria associated with lower and upper respiratory tract infections. Several studies, mostly achieved by serological assays, proposed a role for this bacteria in lung cancer risk. Therefore, this study aimed to evaluate the prevalence of Chlamydia pneuomoniae in fresh lung tissues of a sample of Iraqi patients with lung tumors, utilizing polymerase chain reaction (PCR) technique. . Chlamydia pneumoniae DNA was detected in $86.67 \%$ of samples. Besides, DNA sequencing of $16 \mathrm{~S} r R N A$ gene revealed that our isolate is closely related to Chlamydia pneumoniae TW183 strain. It is concluded that Chlamydia pneumoniae is found in fresh lung tissues of patients, suggesting past infection, reinfection, or persistent form, which may play a role in the development or exacerbation of lung tumors. Our results confirm the high prevalence of this type of bacteria in Iraqi patients.
\end{abstract}

Keywards: Chlamydia pneumoniae; Lung tumors; Lung tissues; PCR; Respiratory tract infections

\section{التحري عن بكتريا الكلاميديا الرئوية في الانسجة الرئوية المستحصلة من مرضى اورام الرئة العراقيين}

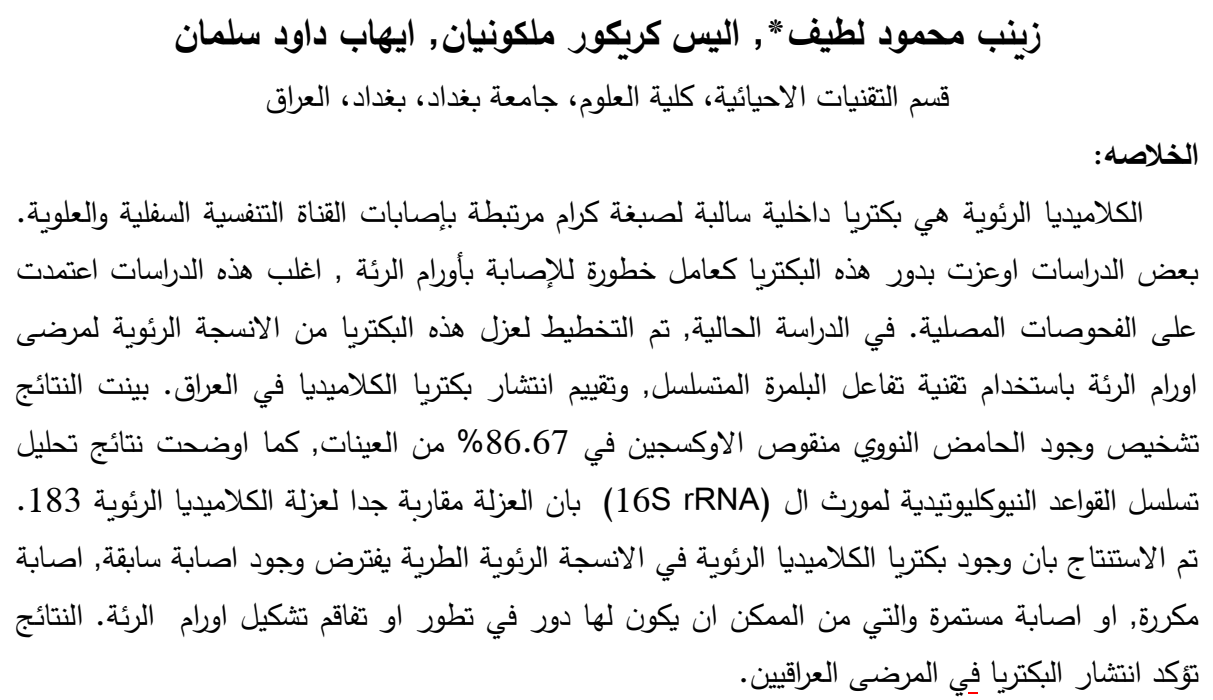

\section{Introduction}

Chlamydia pneumoniae (C. pneumoniae) is an obligate intracellular bacterium that causes upper and lower respiratory tract infections. It is associated with pneumonia, sinusitis, pharyngitis, chronic obstructive pulmonary disease (COPD), and asthma [1]. It is also 
suggested to play a role in lung cancer[2, 3]. C. pneumonia is transmitted via respiratory secretions of symptomatic and asymptomatic individuals and reported to cause 7 to $10 \%$ of community-acquired pneumonia (CAP) among adults, $5 \%$ of bronchitis and sinusitis, and $1 \%$ of pharyngitis cases. Most cases of pneumonia occurring due to $C$. pneumoniae seem to be mild to some extent and self-limited. C. pneumoniae patients with mild symptoms recover within 7-10 days after starting the treatment. The dry cough and general weakness can persist after the disease, slowing the complete remission up to 1 week to 2 months. If neglected, the disease can become worse, leading to severe complications and even mortality [4].

C. pneumoniae undergoes a unique biphasic life cycle. The infectious, but metabolically inactive, elementary body (EB) attaches and enters the epithelium within a vacuole termed inclusion. The EB then matures to a noninfectious metabolically active reticulate body (RB) within $24 \mathrm{~h}$ post-infection. RBs then re-differentiate into EBs in an asynchronous process. At the end of the developmental cycle, after approximately 48-72 h, the EBs are released from the host cell by cell lysis. A multitude of infectious EBs spreads and infects neighboring epithelial cells, initiating the infectious process [5]. The microenvironment undergoes some changes, due to deprivation of essential amino-acids and iron, along with the exposure to interferon-gamma (IFN) and antibiotics [6]. Under these circumstances, the bacteria will halt the production of infectious EBs. This is a persistent state which is characterized by an atypical developmental phenotype termed the aberrant RBs [7]. These persistent forms can remain for a long-time in the host cell and retain their ability to resume the normal developmental cycle when the adverse condition is terminated [8]. The persistence of the RB within cells may occur during long periods due to its ability to hide from the host immune system within inclusion bodies, leading to chronic infection [9].

C. pneumoniae has been found to infect different cell types. In respiratory infections, it initially infects lung epithelial cells and alveolar macrophages. This infection can then spread to the infiltrating immune cells, including monocytes, macrophages, monocyte-derived dendritic cells (DCs), lymphocytes, and neutrophils. In the chronically infected lower respiratory tract, monocytes are proposed to disseminate throughout the body, causing the infection of distal sites [9]. At these sites, the bacteria can infect endothelial cells, smooth muscle cells (SMCs), microglial cells, and neurons. Indeed, C. pneumoniae has long been associated with several chronic inflammatory diseases with prominent effects on public health, mainly atherosclerosis, Alzheimer's disease, and inflammatory arthritis [10-12]

Lung cancer is one of the deadliest and most common malignancies in the world. Several studies have looked for the association of $C$. pneumoniae with lung cancer, although with somewhat varied results. The mechanism by which $C$. pneumonia mediates the development of lung cancer is not well defined. However, the most accepted one is through induction of immune response and further production of inflammatory mediators, which in turn can enhance the production of reactive oxygen species (ROS), resulting in tissue damage, DNA damage or mutations, increased cell turnover, thereby leading to uncontrolled cell proliferation [13]. C. pneumoniae was previously reported to inhibit host cell apoptosis by staurosporine (STS) and TNF- $\alpha$ [14]

The aim of the current study is to detect the prevalence of $C$. pneumonia in lung tissues from patients who are characterized by computerized tomography (CT) scan or chest X-ray to have tumors in their chest and underwent lung biopsy procedure. The polymerase chain reaction (PCR) technique is utilized as a direct assay for the detection of the bacteria in lung tissue biopsies through the $16 S$ rRNA gene. To our knowledge, this is the first attempt worldwide focusing on the detection of $C$. pneumoniae in fresh lung tissues, which makes a sense of approved association between chlamydial infections and lung tumors. 


\section{Methods}

\section{Patients enrolled in the study}

To detect the prevalence of $C$. pneumoniae in lung tissues, a total of thirty $(\mathrm{n}=30)$ lung tumor patients were examined. Those were 21 males and 9 females at ages from 15 to 80 years. Patients' characteristics were further categorized according to histopathology of tumor (malignant or benign) and smoking history, as illustrated in table 1. The study was ethically approved by the Ministry of Health in Iraq (decree order 12304 on 27/3/2019). Samples were collected from Al-Shaheed Ghazi Al-Hareery Hospital for Specialized Surgery/ Medical city office. All patients were informed and consent was obtained before the start of the study.

\section{The polymerase chain reaction assay}

The fresh tissue samples were obtained under aseptic conditions from different parts of the lungs (upper lobe, lower lobe, mediastinal surface). Sections weighing no more than $25 \mathrm{mg}$ were stored in eppendorf tubes $(1.5 \mathrm{ml})$ containing normal saline solution at $-20^{\circ} \mathrm{C}$. For DNA extraction, each frozen biopsy specimen was thawed and crushed with a micro pestle. Genomic DNA was then extracted directly following kit instructions (QIAamp tissue DNA extraction kit, Qiagen). The concentration of the extracted DNA was measured using Qubit 4 fluorometer instrument. DNA samples were stored in the microcentrifuge tube at $-20{ }^{\circ} \mathrm{C}$ until use.

The PCR was performed to detect $C$. pneumoniae in lung biopsies through $16 S$ rRNA gene. This reaction was applied on one set of $16 S$ rRNA primers; Cpn A (5'TGACAACTGTAGAAATACAGC-3') and Cpn B (5'- CGC CTC TCTCCT ATAAAT-3') specific for the 463bp fragment of $16 S r R N A$ gene [15]. Total reaction volume was $25 \mu$, composed of $12.5 \mu \mathrm{l}$ of Q5® high fidelity mastermix, $4 \mu \mathrm{l}$ of DNA sample, $2 \mu \mathrm{l}$ of $10 \mathrm{pmol} / \mu \mathrm{l}$ for each forward and reverse primer, in addition to $4.5 \mu 1$ of free-nuclease water. The same reaction was applied on the positive control DNA sample extracted from $C$. pneuomoniae CWL29 strain (provided kindly by Nabu Scientific Foundation, Iraq). PCR condition was as follows: an initial denaturation at $98^{\circ} \mathrm{C}$ for 1 minute followed by 35 cycles, each consisting of denaturation at $98^{\circ} \mathrm{C}$ for 10 seconds, annealing at $58^{\circ} \mathrm{C}$ for 30 seconds, then elongation at $72^{\circ} \mathrm{C}$ for 30 second, and a final extension step at $72^{\circ} \mathrm{C}$ for 2 minutes.

After amplification, ten microliters of PCR product (mixed with gel loading dye) and DNA ladder (100-1000 bp, NEB®, England) were loaded into the wells of 2\% agarose gel. The voltage of the power supply was mostly fixed at $70 \mathrm{~V}$ for 80 mins. At the end of the run, gel documentation using a GelDoc system (cleaver, USA) with a high-resolution camera was applied to capture the image and analyze the bands.

\section{Sequence analysis of 16S rRNA gene}

For sequence analysis, the sample included $50 \mu 1$ of PCR amplification reaction, containing $25 \mu 1$ of Q5 high-fidelity $\left(\mathrm{NEB}^{\circledR}\right)$ mastermix, $8 \mu \mathrm{l}$ of DNA sample, $4 \mu 1$ (10 pmol/ $\left.\mu \mathrm{l}\right)$ of each primer, and $9 \mu$ l of free-nuclease water. The reaction was conducted under the optimal PCR conditions for this gene, as mentioned previously. About $40 \mu \mathrm{l}$ of the PCR product of positive samples was sent to Macrogen- South Korea for sequencing by Sanger method to define the basic nucleotides sequences for the gene. The results of sequencing were then analyzed by MEGA7 software to construct a phylogenetic tree of pairwise sequence alignment that was used depending on Blast (Basic Local Alignment Search Tool) [16].

\section{Results}

In the present analysis, C. pneumoniae DNA was detected in 26/30 (86.66\%) of lung tissue specimens, whereas only $4 / 30(13.33 \%)$ revealed a negative result. $80.6 \%$ of male samples revealed positive results, while in females, positive results were found in $100 \%$ of samples. The bacterial DNA was found in patients of all age groups $(15-30,80 \% ; 31-50,90 \%$; and $51-$ $80,90 \%)$. A total of $2 / 4(50 \%)$ of the negative samples belonged to the age group of 15 30years. In terms of histopathology, the positive results were observed in $81.8 \%$ of benign 
tumor-derived tissues, while in malignant subjects, positive results were found in $89 \%$ of specimens. According to smoking history, C. pneumoniae DNA was detected in $83.3 \%$ of smokers and $87.5 \%$ of non-smokers (Table 1).

Samples sequencing analysis at $16 \mathrm{~S} r R N A$ gene region, by comparison of the phylogenetic tree, revealed that our isolate is closely related to the non-respiratory $C$. pneumoniae strain TW183 (NC_005043.1), as shown in Figure 2.

Table 1- Characteristics of Iraqi patients with lung tumors enrolled in this study and their PCR results $(\%)$.

\begin{tabular}{|c|c|c|c|}
\hline \multirow{2}{*}{ Characteristics } & Lung tumor patients & \multicolumn{2}{|c|}{ PCR results } \\
\cline { 3 - 4 } & $(\mathbf{n = 3 0 )}$ & positive & negative \\
\hline \multicolumn{4}{|c|}{ Gender } \\
\hline female & $9 / 30(30 \%)$ & $9 / 9(100 \%)$ & $0 / 100(0 \%)$ \\
\hline male & $21 / 30(70 \%)$ & $17 / 21(80.6 \%)$ & $4 / 21(19.04 \%)$ \\
\hline \multicolumn{4}{|c|}{ Age (years) } \\
\hline $15-30$ & $10 / 30(33.3 \%)$ & $8 / 10(80 \%)$ & $2 / 10(20 \%)$ \\
\hline $31-50$ & $10 / 30(33.3 \%)$ & $9 / 10(90 \%)$ & $1 / 10(10 \%)$ \\
\hline $51-80$ & $10 / 30(33.3 \%)$ & $9 / 10(90 \%)$ & $1 / 10(10 \%)$ \\
\hline \multicolumn{5}{|c|}{ Histopathology of tumor } \\
\hline Malignant & $19 / 30(63 \%)$ & $17 / 19(89 \%)$ & $2 / 19(10.5 \%)$ \\
\hline Benign & $11 / 30(36 \%)$ & $9 / 11(81.8 \%)$ & $2 / 11(18.2 \%)$ \\
\hline \multicolumn{5}{|c|}{ Smoking history } \\
\hline Smoker & $12 / 30(40 \%)$ & $10 / 12(83.3 \%)$ & $2 / 12(16.7 \%)$ \\
\hline Non-smoker & $16 / 30(53.3 \%)$ & $14 / 16(87.5 \%)$ & $2 / 16(12.5 \%)$ \\
\hline unknown & $2 / 30(6.67 \%)$ & $2 / 2(100 \%)$ & $0 / 2(0.0 \%)$ \\
\hline
\end{tabular}

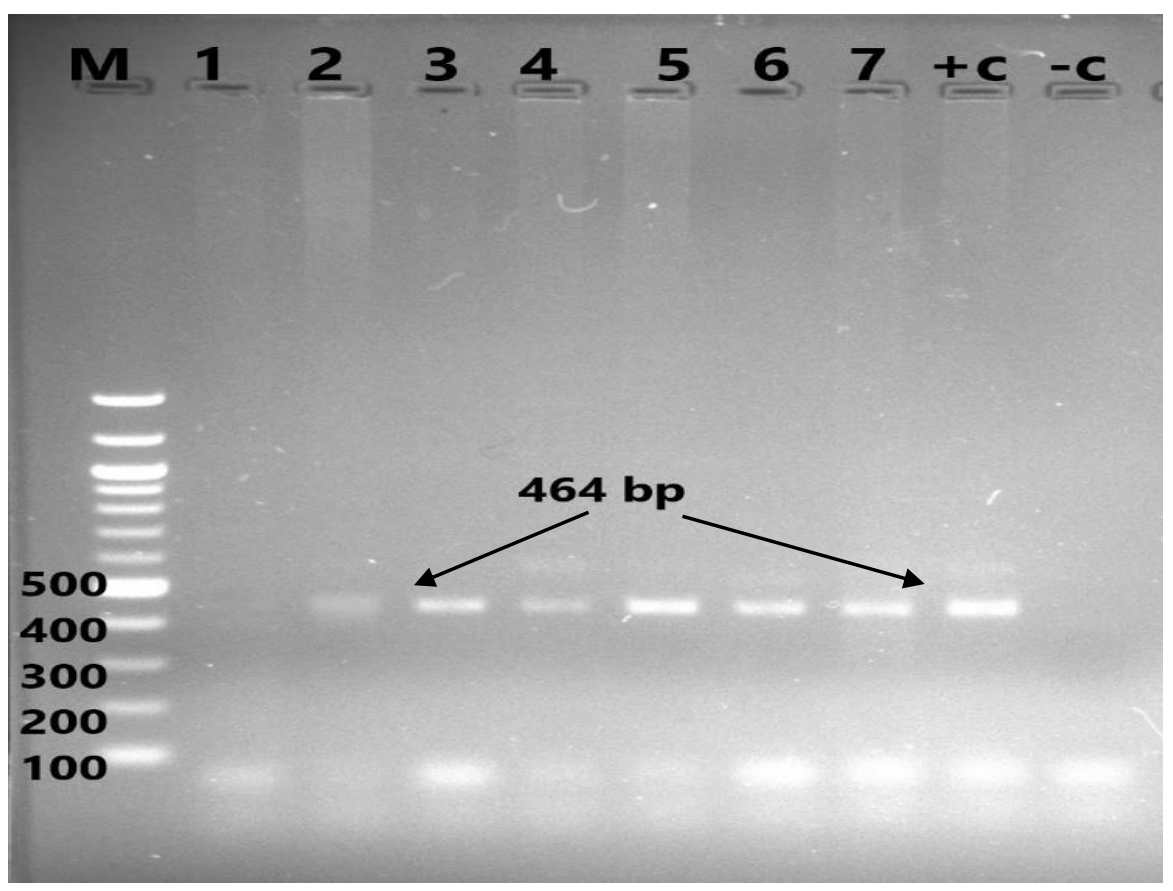

Figure 1- Agarose gel (2\%) stained by RedSafe ${ }^{\circledR}$ for $16 \mathrm{~S} r R N A$ gene with amplification product (464 bp). Lane M: 100bp step marker ladder. Lane (1): negative sample. Lanes 2, 3, 4, 5, 6, and 7: positive samples. Lane +C: Chlamydia pneuomoniae CWL29 strain sample as positive control. Lane -C: negative control. Electrophoresis time of 80 minutes at 70volt. 


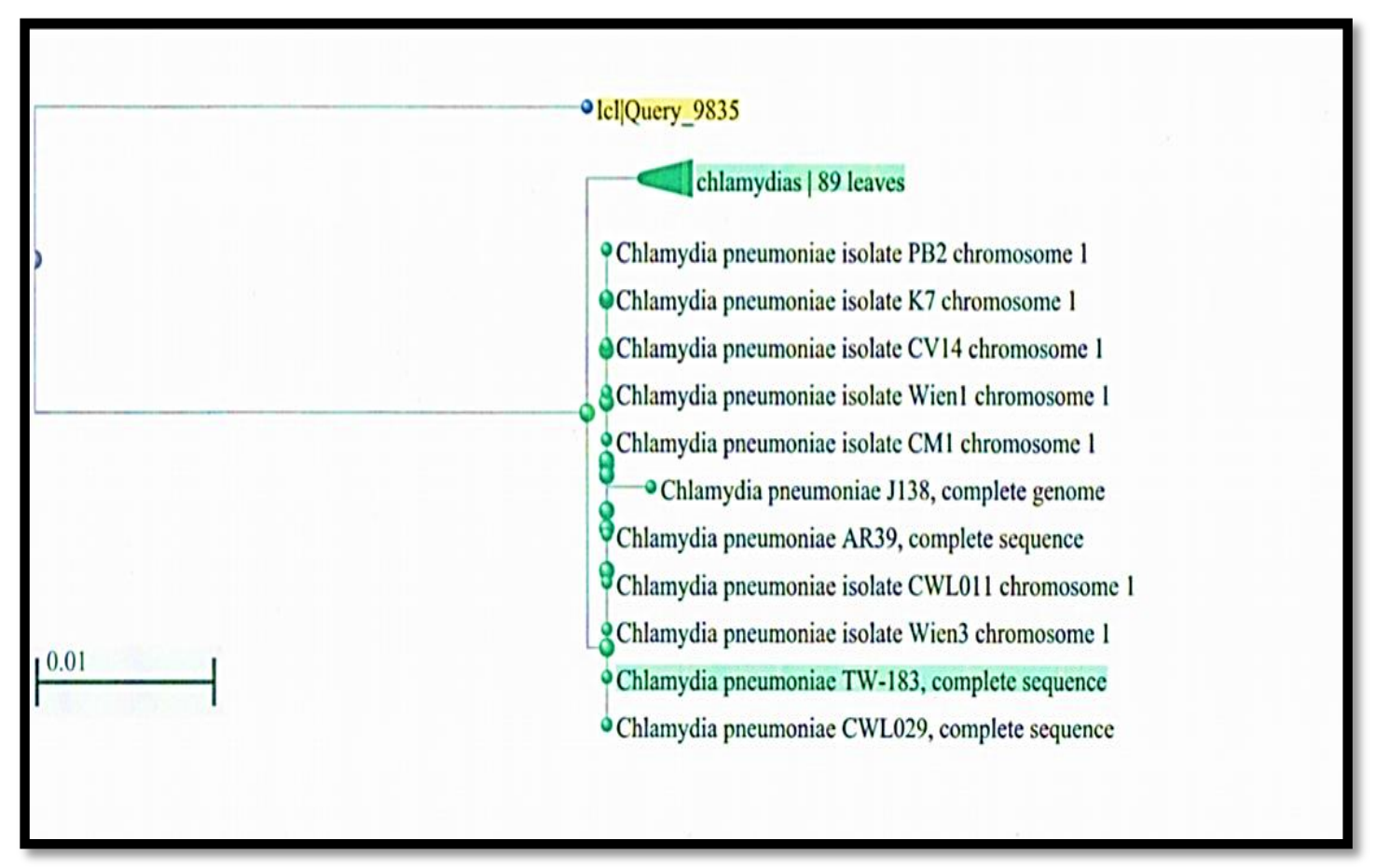

\section{Discussion}

Figure 2-Phylogenetic tree of chlamydias with query_9835.

Chlamydia pneumoniae is a Gram-negative obligate intracellular bacterium that causes a respiratory infection in children and adults, with a high rate in elder individuals. The association between chlamydial infections and the risk of lung cancer is widely reported, although with varying results. However, the majority of these works relied on seroprevalence positivity of C.pneumoniae antibodies using the microimmunoflurescene technique (MIF). Our study achieved by utilizing fresh lung tissues preserved initially in normal saline and deep freezing till analysis by conventional PCR through the $16 S$ rRNA gene. Chlamydia pneumoniae was found in $86.6 \%$ of subjects, which suggests persistent DNA and a further persistent form of infection. Persistent forms are characterized by continuous synthesis of unprocessed 16S rRNA. They are proposed by other workers to be associated with the initiation and exacerbation of chronic inflammatory diseases, in addition to lung cancer [7]. Moreover,, bacterial DNA may indicate repeated or past infections. All the examined females $(100 \%)$ had a chlamydial DNA in the lung tissues, which is a higher incidence than that in males $(80.5 \%)$. According to age, $50 \%$ of the total negative samples were under the age group of 15-30 years. Furthermore, $89 \%$ of malignant tumor and $100 \%$ of benign tumors tissues were positive for $C$. pneumonia. These results suggest an association between the bacterial infection and tumors, regardless of smoking history. The total negative results (13.4\%) could be false-negative; i.e. the biopsy may be resected distantly from the bacterial colonization site. Detection of $C$. pneumoniae in lung tissues from subjects with COPD was achieved by many workers using PCR or immunohistochemical staining. Wu et al., 2000, reported that all archival tissues from COPD subjects who underwent lobectomy for bronchial carcinoma were found to be positively stained for $C$. pneumoniae (100\%). Of these, DNA was detected in only three of six subjects (50\%) using PCR, while in a second group (autopsy), the positive staining was found in $44 \%$ of subjects only [17]. By using nested PCR, $C$. pneumoniae DNA was detected in four out of $31(12.9 \%)$ formalin and HOPE-treated lobectomy lung specimens from COPD patients [18]. C. pneumoniae was also detected in lung tissues derived from patients with advanced emphysema using immunohistochemistry [19]. Chronic inflammatory diseases, other than the respiratory ones, such as atherosclerotic cardiovascular disease, arthritis, Alzheimer's disease, multiple sclerosis, and schizophrenia have been 
suggested to have an association with the persistent bacterial form. The molecular assays are widely used to detect chlamydial DNA in blood, plaques, and tissue specimens derived from patients with chronic cardiovascular and neural diseases [20-22].

Prior to our work, other studies reported that the whole genome and single nucleotide polymorphism (SNP) comparisons of the human strains (AR39, CWL029, J138 and TW183) revealed remarkable similarity in their gene content, arrangement, and nucleotide usage, with 99\% nucleotide identity between human C. pneumoniae strains [23]. Moreover, TW183 appears to form a subgroup branching off from the respiratory strain, $C$. pneumoniae CWL029 [24]. Despite the high prevalence percentage (86.6\%) of chlamydial DNA in our study, we faced challenges in obtaining feasible tissue specimens. In addition, the optimal PCR conditions recommended in the method above were achieved after many attempts of optimization and by using different types of mastermix solutions. However, the difficulties of $C$. pneumoniae detection in other respiratory infections-derived specimens, such as nasopharyngeal swab, sputum, or bronchoalveolar lavage, were reported and atributed to the limited performance of these tests [25].

\section{Conclusions}

The detection of $C$. pneumoniae in fresh lung tissues through $16 S r R N A$ gene suggests past infection, reinfection, or persistent form, which may play a role in the development or exacerbation of lung tumors. However, the exact mechanism is yet to be studied. The present results confirm the prevalence of this type of bacteria in Iraqi patients, while not being taken into consideration by the physicians.

\section{Acknowledgment}

The authors appreciate the efforts presented by the physicians at AL-Shaheed Ghazi ALHareery Hospital, namely, Professor Dr. Waleed Mustafa, Dr. Sabah Noori, Dr. Mumtaz AlNema, Dr. Talaat Refaat, and Dr. Mohammad Mowafaq.

\section{References}

[1] Hammerschlag, M. R.; Kohlhoff, S. A., and Gaydos, C. A. "Chlamydia pneumoniae". Mandell, Douglas, and Bennett's Principles and Practice of Infectious Diseases, 2174, 2015.

[2] Zhan, P.; Suo L. J.; Qian, Q.; Shen, X. K.; Qiu L. X.; Yu, L. K., and Song, Y. "Chlamydia pneumoniae infection and lung cancer risk: a meta-analysis". European Journal of Cancer, vol. 47, no. 5, pp. 742-747, 2011.

[3] Littman, A. J.; Jackson, L. A, and Vaughan, T. L. "Chlamydia pneumoniae and lung cancer: epidemiologic evidence". Cancer Epidemiology and Prevention Biomarkers, vol. 14, no. 4, pp. 773-778, 2005.

[4] Choroszy-Krol, I.; Frej-Mądrzak, M.; Hober, M.; Sarowska, J.; and Jama-Kmiecik, A. "Infections caused by Chlamydophila pneumoniae". Adv. Clin. Exp. Med, vol. 23, no. 1, pp. 123126, 2014.

[5] AbdelRahman, Y. M.; and Belland, R. J. "The chlamydial developmental cycle". FEMS microbiology reviews, vol. 29, no. 5, pp. 949-959, 2005.

[6] Panzetta, M. E.; Valdivia, R. H.; and Saka H. A. "Chlamydia persistence: a survival strategy to evade antimicrobial effects in-vitro and in-vivo". Frontiers in microbiology, vol. 9, pp. 3101, 2018.

[7] Wyrick, P. B. "Chlamydia trachomatis persistence in vitro: an overview". The Journal of infectious diseases, vol. 201, no. (Supplement_2), pp. S88-S95, 2010.

[8] Schoborg, R. V. "Chlamydia persistence-a tool to dissect chlamydia-host interactions". Microbes and infection, vol. 13, no. 7, pp. 649-662, 2011.

[9] Di Pietro, M.; Filardo, S.; Romano, S., and Sessa R. "Chlamydia trachomatis and Chlamydia pneumoniae interaction with the host: Latest advances and future prospective" . Microorganisms, vol. 7, no. 5, pp. 140, 2019.

[10] Campbell, L. A.; and Rosenfeld, M. E. "Infection and atherosclerosis development". Archives of medical research, vol. 46, no. 5, pp. 339-350, 2015. 
[11] Balin, B. J.; Hammond C. J.; Little, C. S.; Hingley, S. T.; Al-Atrache Z.; Appelt D. M.; and Hudson A. P. "Chlamydia pneumoniae: An etiologic agent for late-onset dementia". Frontiers in aging neuroscience, vol.10, pp. 302, 2018.

[12] Carter, J. D.; and Hudson A. P. "Recent advances and future directions in understanding and treating Chlamydia-induced reactive arthritis". Expert review of clinical immunology, vol. 13, no. 3, pp. 197-206, 2017.

[13] Chang, A. H.; and Parsonnet, J. "Role of bacteria in oncogenesis". Clinical microbiology reviews, vol. 23, no. 4, pp. 837-857, 2010.

[14] Rahman, M. A.; Shirai, M.; Aziz, M. A.; Ushirokita, R.; Kubota, S.; Suzuki, H.; and Azuma, Y. "An epistatic effect of apaf-1 and caspase-9 on chlamydial infection". Apoptosis, vol. 20, no. 10, pp. 1271-1280, 2015.

[15] Gaydos, C. A.; Quinn, T. C.; and Eiden, J. J. "Identification of Chlamydia pneumoniae by DNA amplification of the 16S rRNA gene". Journal of clinical microbiology, vol. 30, no. 4, pp. 796800, 1992.

[16] Hall, B. G. "Building phylogenetic trees from molecular data with MEGA". Molecular biology and evolution, vol. 30, no. 5, pp.1229-1235, 2013.

[17] Wu, L.; SKINNER, S. J.; LAMBIE, N.; VUlETIC, J. C.; BLASI, F.; and BLACK, P. N. "Immunohistochemical staining for Chlamydia pneumoniae is increased in lung tissue from subjects with chronic obstructive pulmonary disease". American journal of respiratory and critical care medicine, vol. 162, no. 3, pp. 1148-1151, 2000.

[18] Rupp, J.; Droemann, D.; Goldmann, T.; Zabel, P.; Solbach, W.; Vollmer, E.; and Maass, M. "Alveolar epithelial cells type II are major target cells for C. pneumoniae in chronic but not in acute respiratory infection". FEMS Immunology, and Medical Microbiology, vol. 41, no. 3, pp. 197-203, 2004.

[19] Brandén E; Gnarpe J; Hillerdal G; Orre L; Sköld M., Löfdahl, Hirsh Koyi M, and Tornling G. "Detection of Chlamydia pneumoniae on cytospin preparations from bronchoalveolar lavage in COPD patients and in lung tissue from advanced emphysema" . Int J Chron Obstruct Pulmon Dis.; 2(4):643-650; 2007.

[20] Lantos, I.; Endrész, V.; Virok, D. P.; Szabó, A.; Lu, X.; Mosolygó, T.; and Burián, K. "Chlamydia pneumoniae Infection Exacerbates Atherosclerosis in ApoB100only/LDLR-/Mouse Strain". BioMed research international, 2018.

[21] Eskandarion, M. R.; Nasr, R.; AKBARI EIDGAHI, M. R.;Mozaffari, E.; Norouzi, M., Tabasi, M.; and Gharehdaghi, J. "Prevalence of Chlamydia pneumoniae Infection in Atherosclerotic Plaques of Corpses Referred to Iranian Legal Medicine Organization, Tehran". Journal of Clinical , and Diagnostic Research, vol. 12, no. 6, 2018.

[22] Boelen, E.; Steinbusch, H. W.; Van der Ven, A. J.; Grauls, G.; Bruggeman, C. A.; and Stassen, F. R. "Chlamydia pneumoniae infection of brain cells: an in vitro study". Neurobiology of aging, vol. 28, no. 4, pp. 524-532, 2007.

[23] Rattei, T.; Ott, S.; Gutacker, M.; Rupp, J.; Maass, M.; Schreiber, S.; and Gieffers, J. "Genetic diversity of the obligate intracellular bacterium Chlamydophila pneumoniae by genome-wide analysis of single nucleotide polymorphisms: evidence for highly clonal population structure". BMC genomics, vol. 8, no. 1, pp.355, 2007.

[24] Roulis, E.; Polkinghorne, A.; and Timms, P. "Chlamydia pneumoniae: modern insights into an ancient pathogen". Trends in microbiology, vol. 21, no. 3, pp.120-128, 2013.

[25] Pierce, V. M.; Elkan, M.; Leet, M.; McGowan, K. L.; and Hodinka, R. L. "Comparison of the Idaho Technology FilmArray system to real-time PCR for detection of respiratory pathogens in children". Journal of clinical microbiology, vol. 50, no. 2, pp. 364-371, 2012. 